\title{
Design and Implementation of a Long Range Autonomous Wireless Critical System and its Application in Remote Hydroelectrical Infrastructure (Water Canals) for Risk Prevention and Hazards in Case of Hydraulic Canals Breaks.
}

\author{
Fco. Javier Fernández Huerta ${ }^{1}$, Luis Javier Etayo Pérez ${ }^{2}$, Fco. Javier Alonso Caballero ${ }^{1}$ \\ ${ }^{1}$ KUNAK Technologies S.L. Pol. Ind. Mocholí, Plaza CEIN 5. 31110, Noáin. SPAIN. \\ ${ }^{2}$ Acciona Energía S.A. Av. Ciudad de la Innovación 5, 31621, Sarriguren. SPAIN \\ jfernandez@kunak.es ; luisjavier.etayo.perez@acciona.com ; falonso@kunak.es
}

\begin{abstract}
A new wireless critical system based on Industrial Internet of Things (IIoT) innovations, a combination of different wireless technologies - from mature well known $2 \mathrm{G}$ networks to high performance ultralong range private $169 \mathrm{MHz}$ LoRa network - and a cloud distributed architecture with advanced algorithms is presented to build a critical telemetry system, in this case, applied to real time hydraulic canals monitoring of hydroelectric plants. Thus, sporadic and uncontrolled breaks of hydraulic elements are avoided, decreasing the affection to populations, environment, nearby roads and other infrastructures.
\end{abstract}

For this pilot deployment 6 different hydraulic canals located in remote sites, some of them without cellular coverage, have been monitored with up to 25 measurement points. The wireless remote terminal units (RTU) have been designed with cutting edge lloT electronics, enabling ultra-low power consumption and autonomous energy harvesting operation with $3 \mathrm{~W}$ solar panels; allowing 1-minute sampling time and 5 minutes transmission periods to the cloud, where advanced algorithms, requiring the information of every RTU, update the state and health of the infrastructures in real time.

Key words: 169 MHz LoRa Network, Smart Utility Networks, Hydro Electrical Infrastructures, Autonomous Telemetry Devices, Cloud-based telemetry solution.

\section{Introduction}

ACCIONA SA is a utility company, leader in providing sustainable solutions for infrastructure and renewable energy projects across the world, with $7,254 \mathrm{M} €$ revenues and 37,400 employees. Its offer covers the whole value chain, from design and construction to operation and maintenance, with a presence in more than 65 countries.

KUNAK Technologies SL has designed a disruptive solution to deploy wireless monitoring systems whose ultra-energy efficiency characteristics and end-to-end critical system architecture provide great advantages in isolated, remote and complex environments, guaranteeing the robustness and integrity of 24/7 communications.

ACCIONA is requiring a solution to control more than $300 \mathrm{~km}$ of hydroelectrical infrastructure for risk and hazards prevention in case of hydraulic canals breaks. These canals are used to transport the water from a river or dam, across several kilometers, to the forebay tank in order to gain the higher height difference to the power house (turbine) as possible. Some of these infrastructures were built 100 years ago.

Then, it is necessary to find a solution for fast canals breaks detection; reducing their consequences, minimizing canals affections or even predicting the breakages. This will help develop best practices in maintenance of hydroelectrical infrastructures.

This digitalization process had to solve several challenges (see Table 1) such as gathering the data efficiently in remote scenarios with difficult access, coverage problems and lack of power grid; reliable transportation to Internet; mass storage and Cloud computing availability; advanced alarm generation and ease operation and maintenance of the whole system. 


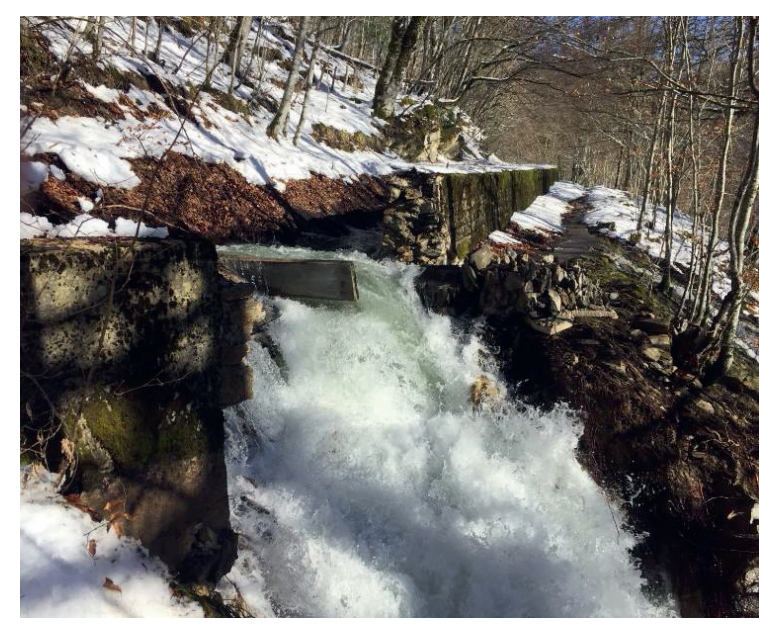

Fig. 1 Water canal break
In previous attempts realized by ACCIONA to face these challenges, electric power and optical fiber lines were installed, resulting in expensive deployments very difficult to maintain.

Also, other data acquisition systems based on mesh and tree wireless sensor network topologies were tested with no success because of their lack of reliability and robustness.

\section{Proposed Solution}

Thus, in the following table the problems to be satisfied by the proposed solution are shown as well as how KUNAK's system solve them and the expected benefits for the customer.

Table 1. Problems, Proposed Solution and Benefits for the Customer.

\begin{tabular}{|c|c|c|}
\hline Problem / Pain & Solution & Benefits \\
\hline $\begin{array}{l}\text { Remote } \\
\text { infrastructures } \\
\text { covering wide } \\
\text { mountainous areas }\end{array}$ & $\begin{array}{l}\text { Wireless devices connected to an } \\
\text { Internet Cloud Platform }\end{array}$ & $\begin{array}{l}\text { Devices can be connected to the } \\
\text { Internet independently of the } \\
\text { technology used, while the information } \\
\text { is centralized in the Cloud }\end{array}$ \\
\hline $\begin{array}{l}\text { No Power - deep } \\
\text { valleys- Low Solar } \\
\text { Radiation area }\end{array}$ & $\begin{array}{c}\text { Ultra-low power consumption } \\
\text { electronics + state of the art energy } \\
\text { harvesting solutions }\end{array}$ & $\begin{array}{l}\text { Autonomous operation without any } \\
\text { additional infrastructure. }\end{array}$ \\
\hline Cost & Wireless and autonomous & $\begin{array}{c}\text { No wire, no civil works, lower CAPEX } \\
\text { and OPEX. }\end{array}$ \\
\hline $\begin{array}{l}\text { Fast \& Easy } \\
\text { installation in } \\
\text { remote areas }\end{array}$ & $\begin{array}{c}\text { Small and light devices, wireless, } \\
\text { autonomous (battery operated), } \\
\text { plug \& play }\end{array}$ & $\begin{array}{l}\text { Easy to transport, install, configure } \\
\text { and operate. Installation ready in less } \\
\text { than } 1 \text { hour. }\end{array}$ \\
\hline $\begin{array}{l}\text { Reach every } \\
\text { infrastructure and } \\
\text { place, no matter its } \\
\text { remote location }\end{array}$ & $\begin{array}{c}\text { Combined wireless solution. } \\
\text { Cellular \& Best-in class long range } \\
\text { wireless sensor network based on } \\
\text { Kunak 169/433/868 MHz LoRa } \\
\text { solution. }\end{array}$ & $\begin{array}{l}\text { Long range: Non-Line-of-Sigth } \\
\text { coverage. (>30Km LoS) } \\
\text { Noise and interferences inmunity.Wide } \\
\text { area coverage in mountainous areas }\end{array}$ \\
\hline $\begin{array}{l}\text { Reliability and } \\
\text { Robustness }\end{array}$ & $\begin{array}{c}\text { IP67 devices with wide temperature } \\
\text { range. } \\
\text { Wireless Critical System } \\
\text { Architecture. }\end{array}$ & $\begin{array}{l}24 / 7 \text { unattended operation. } \\
10 \text { years autonomous operation } \\
\text { without maintenance. }\end{array}$ \\
\hline $\begin{array}{l}\text { Easy Operation \& } \\
\text { Maintenance }\end{array}$ & $\begin{array}{l}\text { Bidirectional system with auto } \\
\text { health checking, alarms and status }\end{array}$ & $\begin{array}{l}\text { Remote tele-control of sensors and } \\
\text { devices. No displacements. }\end{array}$ \\
\hline $\begin{array}{l}\text { Real-time } \\
\text { information on the } \\
\text { health of } \\
\text { infrastructures. }\end{array}$ & $\begin{array}{c}\text { Advanced algorithms running on } \\
\text { the cloud using information from } \\
\text { different sensors, devices and data } \\
\text { sources. }\end{array}$ & $\begin{array}{l}\text { Any virtual condition, situation or } \\
\text { object, can be modeled and presented } \\
\text { to the final user in an ease to use } \\
\text { dashboard. }\end{array}$ \\
\hline $\begin{array}{l}\text { Easy integration } \\
\text { with third party } \\
\text { information } \\
\text { systems }\end{array}$ & $\begin{array}{l}\text { Cloud based solution. } \\
\text { Open API }\end{array}$ & $\begin{array}{c}\text { Easy integration into SCADAs, } \\
\text { information sharing with public bodies } \\
\text { and hydrographic basin organizations. } \\
\text { Access in mobility from Smartphone } \\
\text { Apps. }\end{array}$ \\
\hline
\end{tabular}


In order to satisfy the tight requirements explained before, KUNAK proposed an end to end solution, composed by several elements, building a complete autonomous wireless critical system capable of sensing anywhere through the Internet.

The system has the architecture shown in figure 2 , with the following components:

- Sensors: water level sensors to monitor water height inside the canals.

- Remote Terminal Units (RTUs): Small wireless (2G or LoRa) waterproof devices responsible of reading the sensors and send the information to the cloud. The RTUs are provided with an autonomous power supply system (primary or secondary) for long time operation.
- Gateway: The base station managing the $169 / 433 / 868 \mathrm{MHz}$ LoRa network while maintaining the communication to the Cloud.

- KunakCloud: a cloud-based telemetry \& telecontrol Software capable of managing the sensors, devices and users, store the information, run complex algorithms in real time, provide intelligent alarms, remote configuration and advanced visualization.

- Open API: the open application programming interface allows the solution to interconnect to third party systems and build custom Smartphone Apps for the final customers.

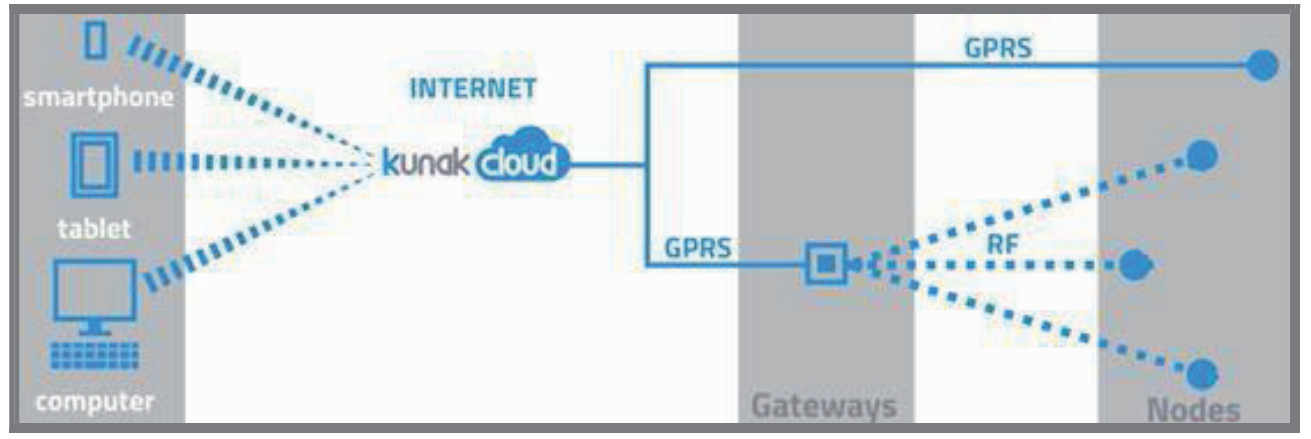

Fig 2. Solution Architecture. Autonomous Nodes using GPRS and Wireless networks based on LoRa modulation are connected to KunakCloud: a Cloud Based Telemetry\&Telecontrol Software.

\section{Detailed System Description}

Sensors: the need of accurately monitoring the water height inside the canals implied the selection of the appropriate sensors capable of measuring the water level with the following considerations:

- Inside the canals, the water is moving, comes from rivers as well as rain falling from the mountains, which make the water to transport mud, leaves...

- Protection from any objet and dirt present in the water as well as to work under extreme environmental conditions.

- Fast response time with low power consumption.

- Low or moderate cost.

For those reasons, radar sensors (high cost, high power consumption), sensors based on conductive and capacitive effects (mud and dirt can influence their readings [1]) and buoys and floats (moving parts that can be obstructed), were discarded. Thus, hydrostatic pressure sensors, particularly the MA-403 [2], were selected because of their fast response, low power consumption, accuracy and reliability. Also, two ultrasonic sensors from Matbotix (MB7369) [3], were tested and installed next to a hydrostatic pressure sensor to compare both sensors readings over long periods of time and different environmental conditions. Both sensors selected are temperature compensated and the RTUs oversample, average and filter the readings to maximize the accuracy of measurements. One reading is taken every minute to have maximum temporal resolution in this application.

RTUs: cutting edge IloT technologies were used to design ultra-low power consumption (few $\mu \mathrm{A}$ in sleep mode) devices capable of measuring the sensors, provide independent power supplies from 5 to $19 \mathrm{Vdc}$ to them, analyze the readings, store them and transmit, using different wireless technologies, to the cloud. The design has two different power supply systems to guarantee the autonomous 
operation depending on the final installation site.

- Primary Batteries: devices which were installed underground or in places with no direct sunlight exposure use long life $7.2 \mathrm{~V}, 14 \mathrm{Ah}$ lithium primary batteries. These units where configured to send the information to the cloud every 15 minutes to guarantee at test 1-year of autonomous operation.

- Secondary Batteries: where small solar panels of $3.5 \mathrm{~W}(6 \mathrm{~V})$ can be installed, the units are provided with $3,7 \mathrm{~V}, 10 \mathrm{Ah}$ $\mathrm{Li}$-Ion rechargeable batteries. This capacity guarantee at least 4 weeks of autonomous operation without solar charge sending the information to the cloud every 5 minutes. The RTUs are provided with power path and manage the solar charge to avoid batteries overcharge and deep discharge, enhancing batteries life up to 5 years of continuous operation.

The RTUs has different input and output ports: analog (4-20mA, 0-10V), digital (total or partial pulse counters, frequency meters up to $4 \mathrm{KHz}$, ON/OFF state), Modbus RTU-485, relays, etc... which can be easily configured from a computer software enabling their calibration during on field installation.

The electronics and batteries are encapsulated inside $12 \times 12 \times 9 \mathrm{~cm} \quad$ IP67 polycarbonate enclosures, with IP68 connectors for the sensors, antenna and solar panel. Also, a metallic roof is provided with the system to ease the mast-mount installation, to provide a support for the solar panel and to protect the enclosure from overheating under strong solar radiation during summer, which could reduce the battery life. Finally, external high gain antennas are used to enhance signal strength in remote areas (figures 6 and 7).

Wireless Communications: in order to maintain a continuous bi-directional connection of sensors and RTUs to the cloud, two different wireless technologies have been used. In most of the required monitoring places, even in mountainous valleys, a reliable $2 G$ connection was present which allowed using these cellular networks for data transmission directly to the cloud.

However, there were some infrastructures and areas not covered by any kind of cellular network, which was an enormous challenge for sensing. Satellite technologies were discarded because its cost and power consumption. That's why, the design of an ultra-long-range radio frequency sensor network capable of maintaining reliable communications in rural environments with abrupt orography was required to complement the whole system solution and fulfil the customer needs.

Thereby, different sensor networks topologies were evaluated. Mesh and tree topologies (Fig.3) can provide the highest coverage range since each device extend the network by "jumping" the information from one device to another. However, if any router node experiments any problem, the whole network will suffer from downtime, not fulfilling the reliable and robustness requirements. The probability of network failures increases with each jump. In fact, in the past, ACCIONA carried out tests with these network topologies undergoing these reliability problems. Besides, as the information must jump across the network, the traffic of the whole system increases as well as its power consumption. Therefore, this kind of networks can suffer from higher duty cycle restrictions, higher latency and much higher power consumption.

For all these reasons, mesh and tree topologies were not considered for high reliability, low latency and low power consumption KUNAK's system architecture in remote scenarios.

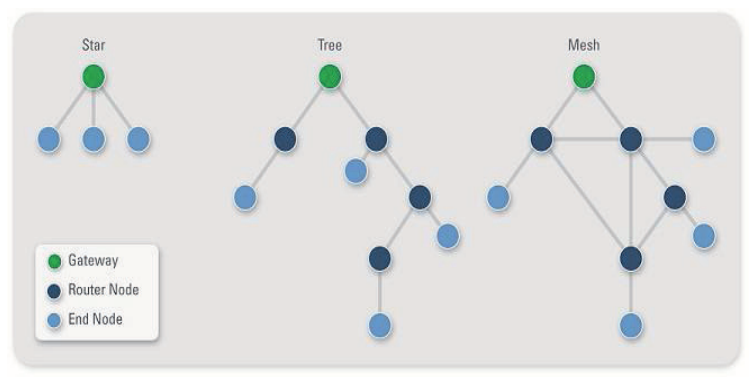

Fig 3. Wireless Sensor Network Topologies.

Star networks are the most reliable as just one point of failure exists, the gateway, that could be redundant to increase robustness in critical applications. Up to date, the main problem of this topology was its coverage since all the end nodes must be within the range of the Gateway and current available modulation techniques within the ISM (industrial, scientific and medical) frequencies (2400/868/433/169 MHz) didn't allow long range reliable communications distances of more than few kilometers Line-of-Sight (LoS) and hundreds of meters Non-Line-of Sight (NLoS).

However, new revolutionary modulation techniques in Sub-GHz bands have appeared in the last few years enhancing dramatically the overall performance of wireless networks. Traditional FSK, spread spectrum like LoRa (Long Range, Semtech Trademark), and ultra- 
narrowband technologies like Sigfox are available in the Sub-GHz ISM bands.

Some of these technologies, like Sigfox [5], are only present in the $868 / 900 \mathrm{MHz}$ band which is a limitation for radio propagation in rural mountainous areas (as shown in figure 8). FSK and LoRa Modulation cover the whole Sub-GHz ISM bands, but the outstanding characteristics in terms of link margin; noise and interference immunity; multipath, fading and doppler resistance; and sensitivity [4] of LoRa modulation, makes this technology ideal for ultra-long range wireless communications requiring high reliability and robustness.

The LoRa Alliance [6] develops and promotes the LoRaWAN open standard to enable large scale Low Power Wide Area Networks (LPWAN), based on LoRa modulation, guarantying interoperability between manufacturers. This standard has been mainly designed for large scale networks with thousands of battery operated devices transmitting very few information a day (IoT devices, smart meters). That's why, the standard can't include all the LoRa and FSK modulation schemes since it is focused on high capacity networks excluding very high and very low data rates. Besides, the LoRaWAN is defined for the $868 / 915 \mathrm{MHz}$ band, which will present problems in applications requiring NLoS communications (hills, mountains, valleys, etc...), excluding other Sub-GHz ISM bands very interesting for remote telemetry systems.

In order to satisfy the requirements of ultra-long range, low power, NLoS telemetry applications, KUNAK has designed a wireless critical system using the LoRa modulation in the $169 / 433 \mathrm{MHz}$ band. The system can also be configured to work in the $868 \mathrm{MHz}$ band for medium range or high throughput, low latency applications (not covered by the LoRaWAN standard: LoRa@ 37.5Kbps, FSK@300Kbps).

Thus, it has been designed a modem based on the SX1276 transceiver [7] with an ultra-low power Cortex M4 microcontroller running a proprietary stack to set star networks with bidirectional confirmed communications. The modem can be configured through a host microcontroller at any available modulation scheme in the SX1276 transceiver allowing sensitivities down to $-149 \mathrm{dBm}$ (18bps), high data rates of $300 \mathrm{Kbps}$, output power up to $27 \mathrm{dBm}$ and different frequency bands $(169 / 433 / 868 \mathrm{MHz})$. Also, interesting features such as Listen Before Talk (LBT), statistics (PER, duty cycle...), as well as automatic acknowledgements are included within the stack to guarantee packet delivery, network status and health, reduce errors, retransmissions and power consumption.

Finally, a Gateway has been designed to centralize the end nodes information, built the start network and send the information to the cloud. This device is based on an embedded Linux platform, running the proprietary gateway stack to manage the previous LoRa modems, with a $4 \mathrm{G} / 3 \mathrm{G} / 2 \mathrm{G}$ modem and ethernet connection; everything encapsulated in IP67 enclosure. The gateway requires continuous power supply since no battery back-up is included in this first version.

KunakCloud: a cloud-based platform, hosted on Amazon Web Services (AWS), has been designed, with the architecture showed in figure 4 , to manage the devices and sensors, store the information and check the overall system performance and health; from anywhere with Internet access.

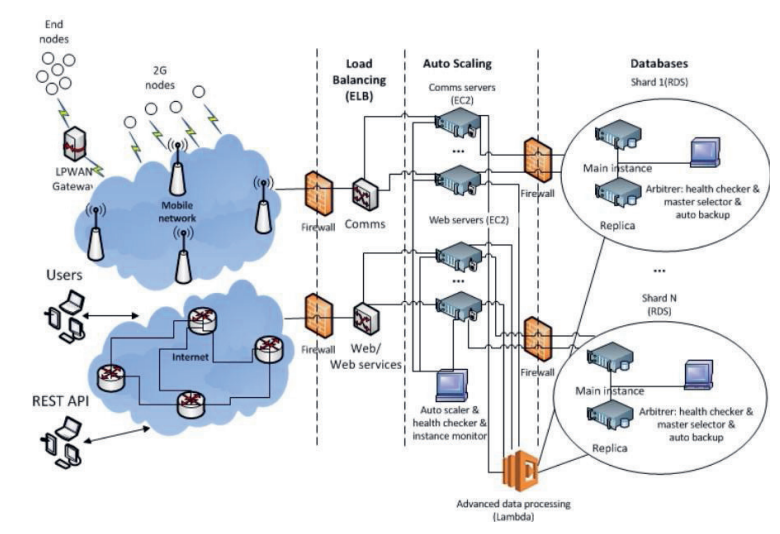

Fig 4: Cloud-platform architecture.

The platform is also responsible of data integrity and interoperability, abstracting the users from any technology involved in the whole system. That is, the user interacts with sensors, devices and configurations such as in a wired system, leaving to KunakCloud the whole responsibility of managing the communications, information, configurations... or automatically recover from any error in communications or servers. RTUs and Gateways has also been designed to automatically recover from any fault, store the information if the communications fail and finally guarantee the delivery of the information to the cloud.

So, all the firmware and software involved in the system is able to handle any errors that may occur and even manage its own recovery. KunakCloud monitors all the control variables, making decisions depending on these, in order to guarantee its integrity based on the boundary conditions. 
Thanks to the data model and cloud architecture, new virtual objects, conditions, rules, etc, can be created to model any desired situation with a combination of real time information from different RTUs, external data and advanced calculations. Thus, real time algorithms are running in the cloud to supervise the desired situations. This is used in this project to model the whole hydroelectrical infrastructure and water canals and create intelligent alarms.

\section{Field Deployment}

The monitoring of six different hydroelectrical infrastructures located in Spain was required within this first pilot deployment, five of them in the Pyrenees and another one in Cantabria. For each infrastructure a deep study was carried out to detect critical points and sections, all the way long of the water canals, where a breakage could produce any risk to human beings, civil works, roads, villages... resulting in 23 hazard points to be monitored.

All these points have access to $2 \mathrm{G}$ cellular coverage instead of 3 points of the Betolegui Infrastructure, located in the area of Selva de Irati (known for being the second largest beechfir forest in Europe). Therefore, 20 points were monitored with $2 \mathrm{G}$ communications RTUs, and a LoRa star network was deployed in Betolegui to reach this challenging area.

Sensors: Every monitoring point was equipped with a hydrostatic sensor, as previously explained. All of them were installed protected with a PVC tube inside the water canal, as shown in figure 3 . In order to evaluate the longtime performance on field of ultrasonic sensors [2], two points were also equipped with this fast response sensors to measure the distance to the water.

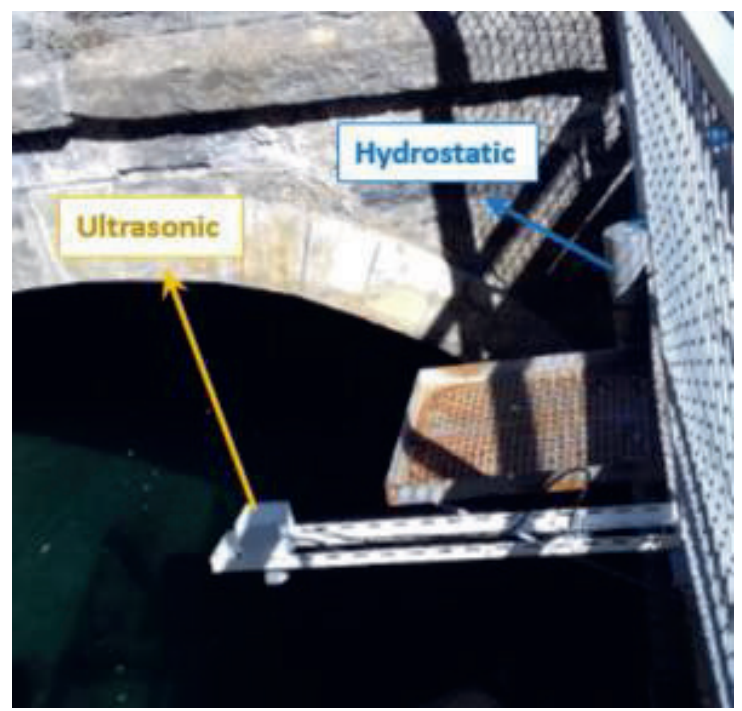

Fig 5. Hydrostatic and ultrasonic sensors installation.
Once the sensors were installed, the operator used the Software Configuration Tool, provided with the RTUs, to configure the sensor's power supply source to one second pre-heating time and to calibrate the $4-20 \mathrm{~mA}$ sensor output to give the absolute water height inside the canal. Sensor readings were configured at 1-minute sampling interval. In the case of ultrasonic sensor, 2 seconds pre-heating time was used and the pulse width output was read and converted to distance, after being averaged and filtered, by the RTU.

2G Deployments: as mentioned before, 20 points have $2 \mathrm{G}$ cellular coverage, 17 of them use solar panels and just 3 of them primary batteries. All of them were installed on the top of 3 meters mast, with $9 \mathrm{dBi}$ Yagi directional antenna (figure 6). Installation of those points were easy and fast without any previous coverage study since it was known the existence of cellular signal. On field, the Software Configuration Tool was used to point the antennas to maximize the received signal.

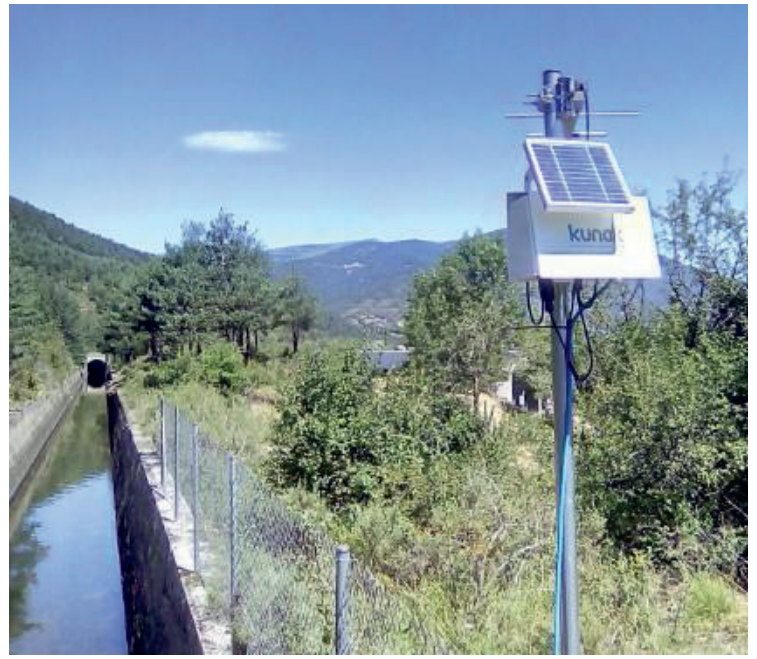

Figure 6: 2 G RTUs with $3.5 \mathrm{~W}$ installed on the top of a 3 meters mast on a water canal side.

LoRa Deployment: in the case of Betolegui infrastructure, a radio coverage study was performed to choose the best frequency and radio configurations and check the viability of this wireless network over the orography.

In the area, a spot with $4 \mathrm{G} / 2 \mathrm{G}$ cellular signal and power grid access was located around the middle of the water canal course which could be a good point for the Gateway installation. So, the coverage study started from this point.

First of all, a LoS analysis was realized with the tool included in google earth, checking that there wasn't LoS with any of the monitoring points (Fig. 7). So, the use of $2.4 \mathrm{GHz}$ ISM frequency band was impossible with star 
networks, almost discarding the use of 868 $\mathrm{MHz}$ band without needing any previous study.

However, a simulation with the LoRa System parameters at 868, 433 and $169 \mathrm{MHz}$ was carried out, using the RadioMobile [8] software, to have an approximate performance estimation before field tests. Thereby, it was checked, as shown in figure 8 , that the $868 \mathrm{MHz}$ frequency didn't reach almost any critical point in the infrastructure (coverage area in black color); getting an appreciable improvement with the $433 \mathrm{MHz}$ band (extended coverage in red). However, some points were still not reached or near the sensitivity values. Finally, thanks to the higher output power available in the $169 \mathrm{MHz}$ band and its better propagation behavior, it was possible to provide a reliable coverage to the whole valley as well as most of the places in $100 \mathrm{Km}^{2}$ (extended coverage from previous frequencies in yellow color).

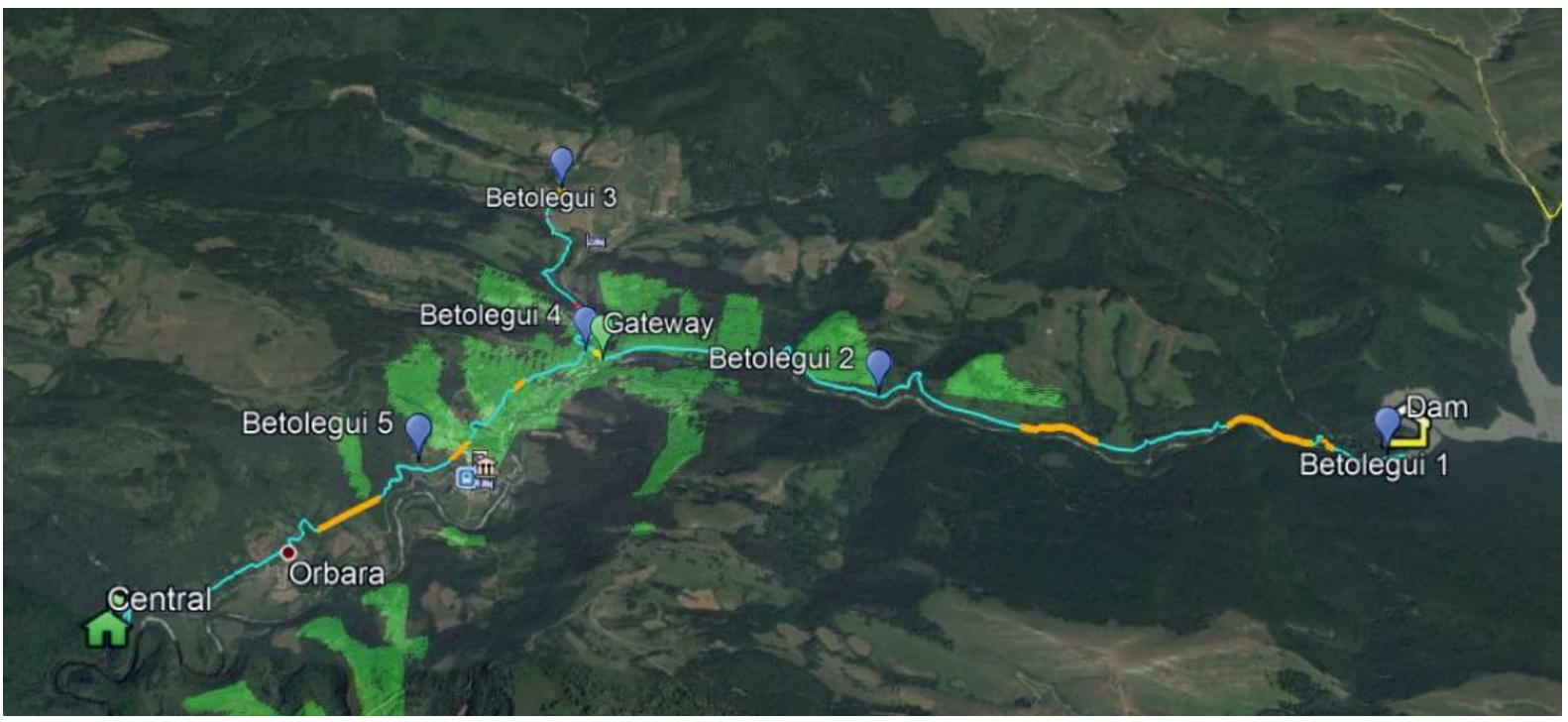

Fig 7. Line of Sight analysis from the Gateway point of view. The water canal and every critical point to be monitored is indicated on the map as Betolegui 1,2,3,4,5. Also the dam and the hydroelectrical plant ("Central").

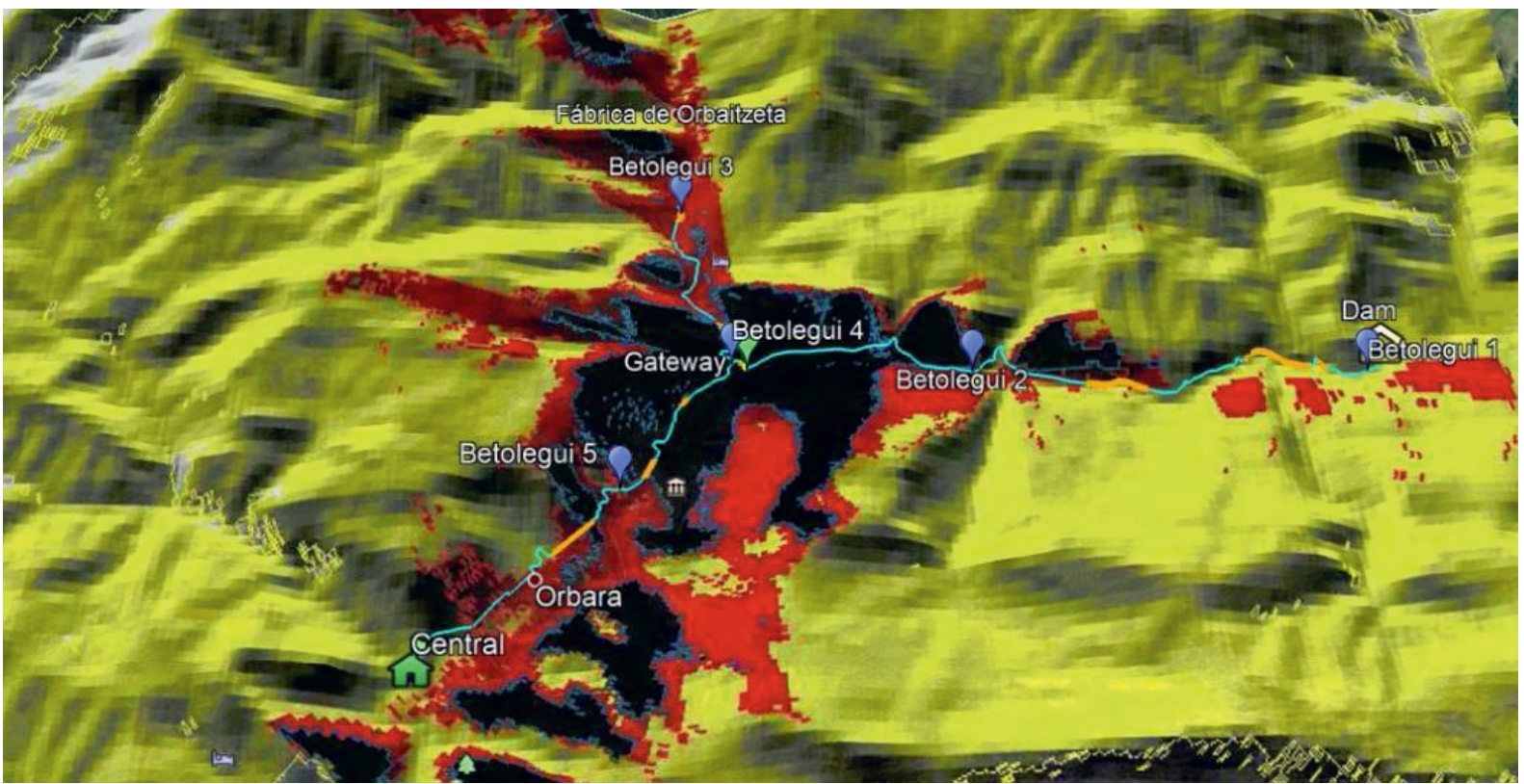

Fig 8: Areas with signal levels above sensitivity $(-137 \mathrm{dBm})$ for three different ISM Sub-GHz frequency bands and output EIRP: $14 \mathrm{dBm}$ for $868 \mathrm{MHz}$ (black), $433 \mathrm{MHz}$ (black+red), $27 \mathrm{dBm}$ for $169 \mathrm{MHz}$ (black+red+yellow)

That's why, it was decided to use the $169 \mathrm{MHz}$ band, with an output EIRP of $27 \mathrm{dBm}$ and $41.7 \mathrm{KHz}$ bandwidth, to fulfill the European directive requirements. A spreading factor of 10 , to have initially a $-137 \mathrm{dBm}$ sensitivity at
$325 b p s$, was chosen to reach the highest link margin as possible without compromising the duty cycle restrictions as just very few devices were going to transmit data once every 5 minutes, corresponding to less than $1 \%$ duty 
cycle, even with retransmissions under communications failures.

After that, a deeper analysis of every point to point radio link was realized with the same software. Here it is only presented the worstcase point, Betolegui 1 , at $4.8 \mathrm{Km}$ distance from the Gateway, where the NLoS scenario can be clearly appreciated in Fig 9. An estimation of $-114 \mathrm{dBm}$ received signal was calculated by the Software. The other points were expected to receive around $-100 \mathrm{dBm}$.

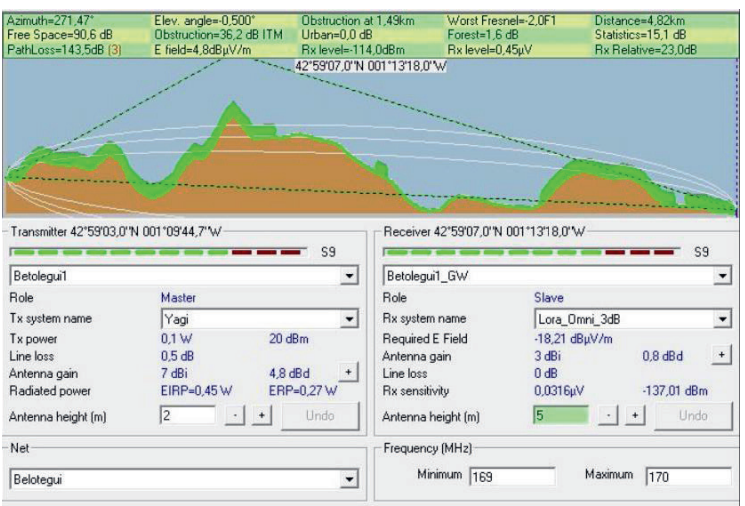

Fig 9. Betolegui 1 to Gateway Radio Link Analysis.

Therefore, it was decided to deploy the LoRa Wireless Sensor Network with the same configuration than the simulations, to have at least $20 \mathrm{~dB}$ link margin that could hold up any unforeseen once installed. The RTUs were installed with different antennas, in order to test on field their influence in the performance of the system and check the coincidence with the simulations. The gateway was installed with a $3 \mathrm{~dB}$ omni-directional antenna, Betolegui 1 with a directional $7 \mathrm{~dB}$ Yagi antenna (Fig. 10) and the other points with $2 \mathrm{~dB}$ (Fig. 11) and $0 \mathrm{~dB}$ omnidirectional antennas.

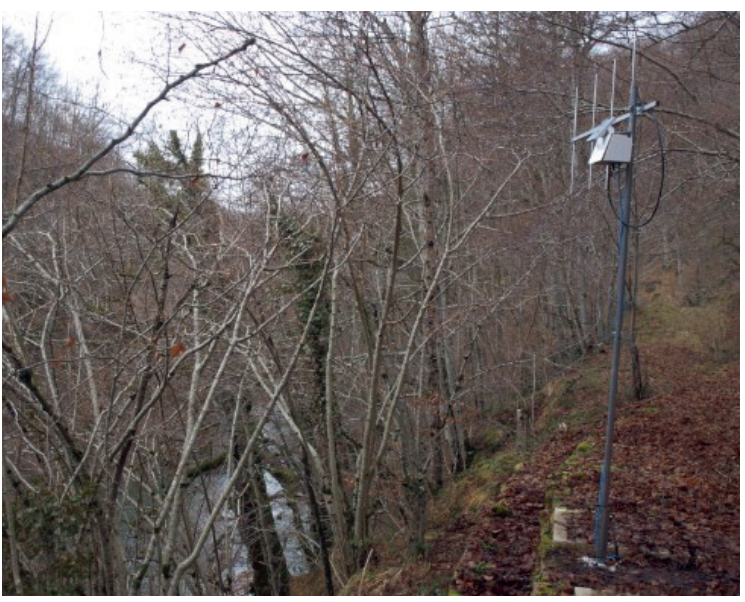

Fig 10. Betolegui 1: 7dB directional Yagi Antenna

The same Software Configuration Tool was used to point the antenna towards the Gateway, and configure the sensors as previously explained.
Cloud-based platform: With all the information being received in a reliable way in the cloud, two different software customizations have been realized:

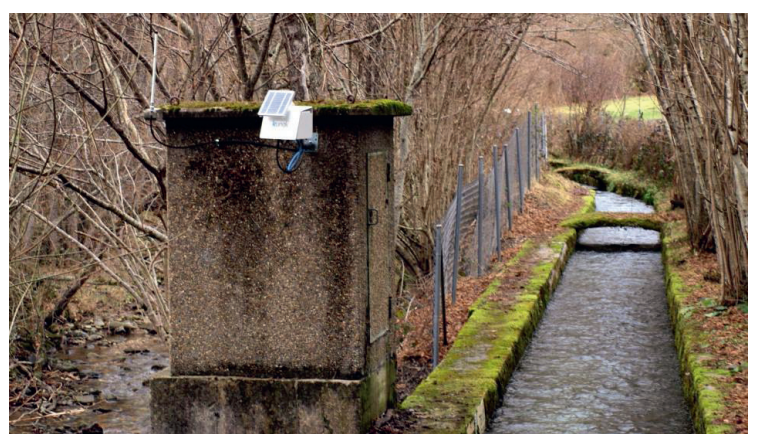

Fig. 11: Betolegui 3: OdB omnidirectional antenna.

1. Definition and design of virtual objects (water canals), advanced algorithms, conditions and alarms to model the different infrastructures and supervise their status and health.

2. Specific dashboard for the easy visualization of the water canals status, their parameters and alarms (Fig 12).

In order to automatically supervise the infrastructures health, each canal was split into different sections by using critical checkpoints where the RTUs were installed. Checkpoints were characterized by a series of civil engineering parameters, used as input to calculate flow levels and alarms.

Flow sensors were virtually created based on relations between the civil engineering parameters and the water level measurements. For every water level sensor there was a virtual flow level sensor.

Alarms were triggered on a time basis relation between the flow levels of a checkpoint and the previous one. If a checkpoint has a current flow level higher than the water flow level of the previous one some time ago, it means a possible partial break or partial break. In a similar way, lower level means a possible obstruction or obstruction.

Using these advanced algorithms, it's possible to identify an alarm with a bounded resolution both in time and space.

Thanks to the cloud architecture and its anywhere accessibility, operators could access on site to the data measured by the RTUs, using a Smartphone App, to check the sensor readings, health and status of the RTUs before leaving the installation site. Also, this App helps them to access the information in real time during maintenance works or in case of alarms. 


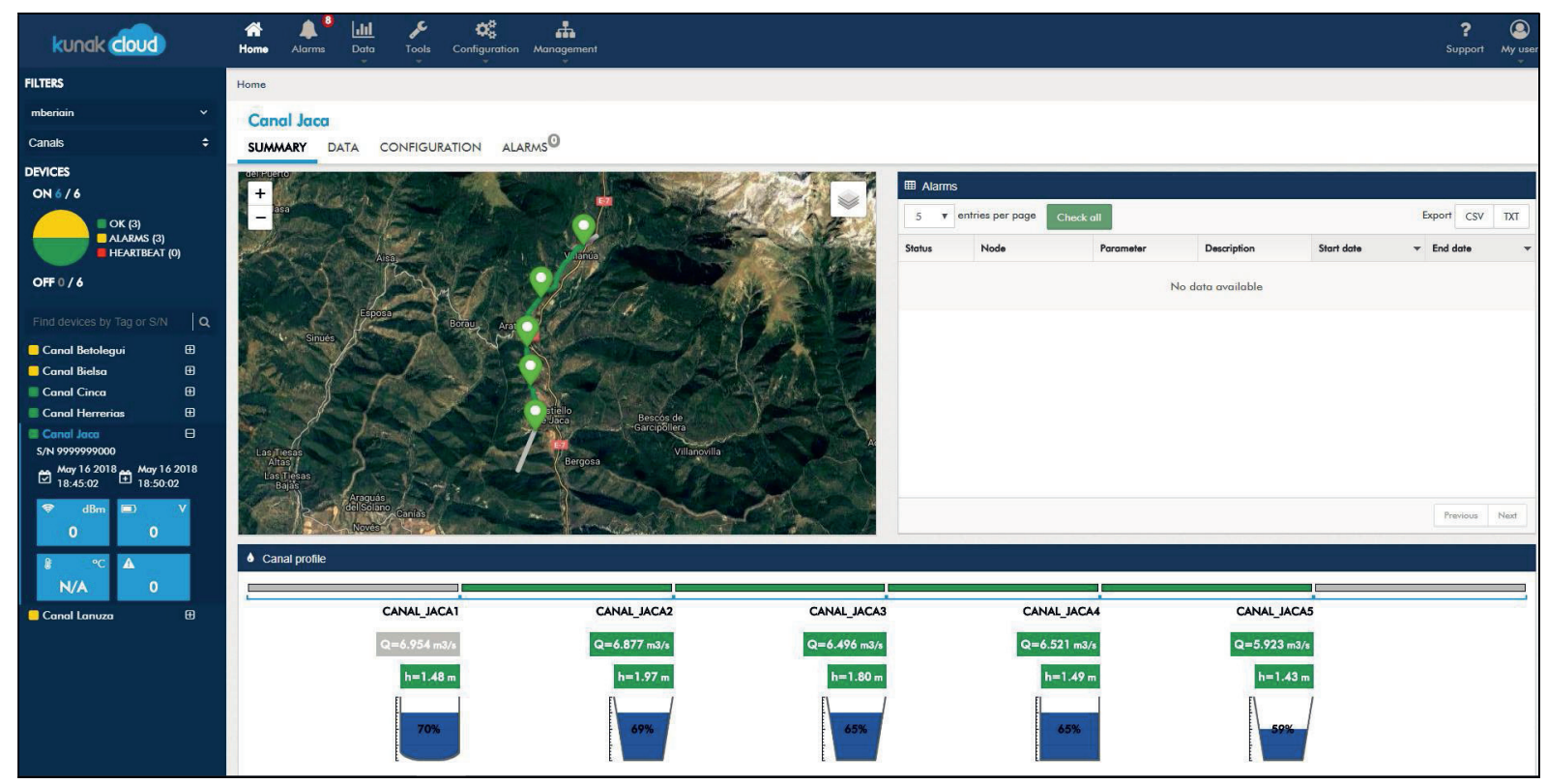

Fig. 12. Customized dashboard visualization. Every critical point is located on the map, dividing the different sections of the canal and reporting a water level and flow values.

\section{Field Results}

After 10 months continuous operation from the field deployment, the system has shown high reliability and robustness with no fails.

Sensors: hydrostatic pressure sensors have demonstrated great performance on field, with high accuracy and reliability (this application just required $1 \%$ accuracy). Comparing to the ultrasonic sensors, the field results show a correlation $R^{\wedge} 2=0.99$ with very similar accuracy over the whole environmental conditions $(-15$ to $+40^{\circ} \mathrm{C}$ ). However, in presence of heavy rain, the ultrasonic sensors have shown an increased noise about $1 \%$ of the reading, that could be reduced with better filtering and signal processing. Sporadic spikes in the ultrasonic sensor reading have also been appreciated which could also be filtered by improved signal processing.

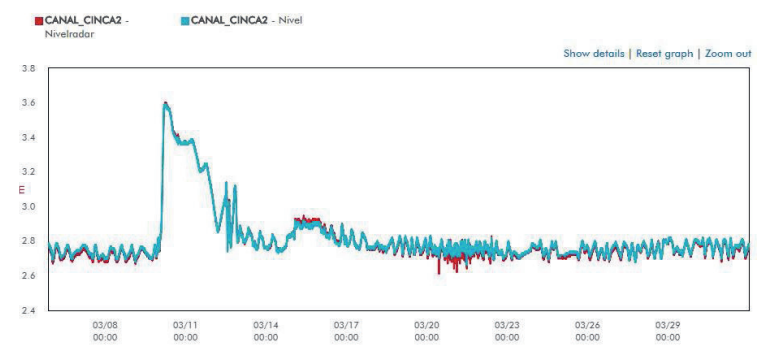

Fig. 13. Hydrostatic vs ultrasonic sensors.

2G Communications: after 10 months operation time, the $2 \mathrm{G}$ communications have demonstrated to be very reliable, even in rural environments, with $99 \%$ successful communications every 5 minutes (within no more than 1-minute delay). Most of failed communications were solved in the next 5 minutes period with just $0,015 \%$ communications problems lasting more than 15 minutes (normally less than 1 hour). Most of these communications problems were caused by the base stations that sometimes are battery operated in rural environments, experiencing in one situation 8h continuous downtime. However, the whole information during communications problems was stored in the RTUs and finally transmitted to the cloud once the communication was reestablished.

RTUs: devices have been working from $-15^{\circ} \mathrm{C}$ to $+40^{\circ} \mathrm{C}$ without any problem. Batteries have shown a great performance even on cold, being able to charge below $0^{\circ} \mathrm{C}$, achieving a capacity greater than $60 \%$ all the time for most of the RTUs.

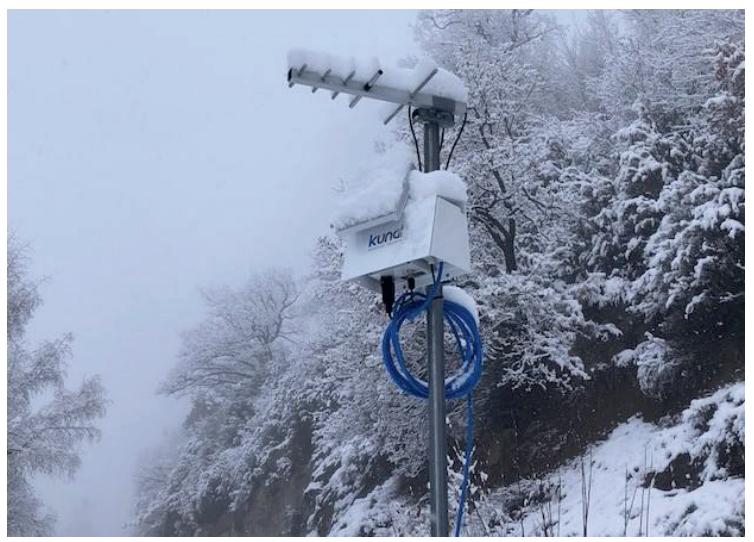

Fig.14. RTU after a snowstorm.

LoRa Communications: the wireless sensor network designed has been proven on field, achieving great performance under every environmental conditions. Simulations have shown to be very reliable in $169 \mathrm{MHz}$ as the 
average signal received by the gateway from Betolegui 1 is $-113 \mathrm{~dB}(-114 \mathrm{dBm}$ simulated) and $-110 \mathrm{dBm}$ for the other points (10dB worse than expected). This difference could be produced by the antennas installed, resulting in a much better and stable performance of the Yagi antenna than the omnidirectional ones (as expected). Also, the RTU with the Yagi antenna has maintained the same received signal from installation while the RTUs with omnidirectional antennas have shown a deterioration in the received signal of around $10 \mathrm{~dB}$.

Hydroelectrical infrastructures: Having accurate information about the water level inside the canals every few minutes provides a great value, since it allows to have a complete control of the evolution of the water level along the whole canal enabling alarms detection when there are unusual levels. Thereby, previously unknown events, like level drops due to dirt or other actions that occur upstream the beginning of the canal, has been detected very easily (Fig. 15)

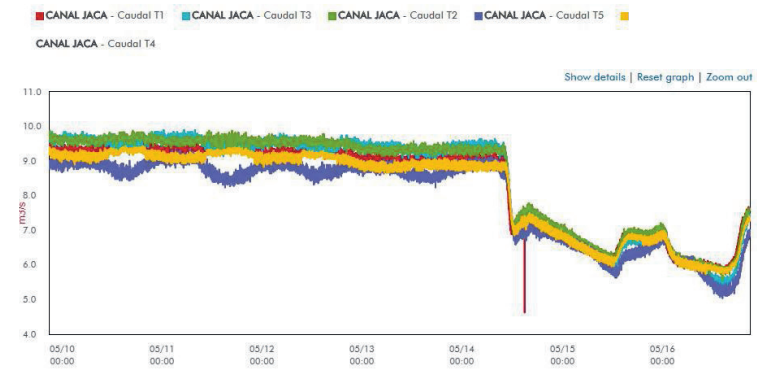

Fig.15. Flow fluctuations in a water canal.

\section{Conclusions}

Conclusions are presented from the system performance point of view and from the infrastructure digitization results.

System Performance: having a distributed cloud telemetry platform facilitates the deployment of RTUs, sensors and loT devices anywhere on earth, using Internet as a virtual communications channel that can be extended wirelessly through innovative LPWAN. The system has been globally designed to add very easily new devices on demand, no matter what their location are.

System reliability and robustness has been proven in this deployment, enabling real time information with autonomous RTUs as if it was a wired solution.

Low power consumption ultrasonic sensor has been tested on field with very good results comparing to hydrostatic sensors. Thus, they offer an alternative for applications where 1-3\% accuracy is allowed.
Finally, a new multi-band LoRa star network has been designed and tested at $169 \mathrm{MHz}$ on field, achieving incredible performance in terms of coverage and reliability, reaching remote locations where before it was not possible to monitor. The use of Yagi antennas is recommended for these deployments, resulting in higher received signal stability reaching similar values than those simulated with RadioMobile.

Hydroelectrical infrastructures digitization: Before this project, ACCIONA didn't have the possibility of gathering real-time data from difficult access isolated points, losing the control of the health and events in the canals.

Thanks to this project, the operations have been digitized enabling data-informed decisions with real-time intelligence.

Unknown behaviors of the water canal levels have been evidenced in parts with non-uniform geometries as well as variations in the inflow rate due to upstream affections. But most important, the high frequency of data has allowed to generate alarms for the early anomalies detection in the cloud and hence the reduction of affections by rapid action.

Overall, the presented solution implements real operational intelligence turning real-time data into actionable results; with an easy to deploy and operate software solution that connects sensor-based data, structures, and people to generate real-time, geo-located insights.

Acknowledgements: The authors wish to thank the whole KUNAK Team for their commitment and work, specially Mikel Beriáin and Asier Galech, for their collaboration with the cloud platform and the LoRa gateway design, and Jacopo Soffritti, for his help with the simulations. Also, the whole ACCIONA team for their support and professionalism on field installation.

\section{References}

[1] Instrument Engineers' Handbook, Fourth Edition, Volume One.

[2] Datasheet MA-403: http://www.inst-apli.com/

[3] Datasheet MB7369: https://www.maxbotix.com/

[4] https://www.semtech.com/uploads/documents/an 1200.22.pdf

[5] https://www.sigfox.com/en/sigfox-iot-technologyoverview

[6] https://LoRa-alliance.org/

[7] https://www.semtech.com/products/wirelessif/LoRa-transceivers/SX1276

[8] http://www.ve2dbe.com/english1.html 